\title{
MicroRNA-494 promotes the proliferation and migration of human glioma cancer cells through the protein kinase $B$ /mechanistic target of rapamycin pathway by phosphatase and tensin homolog expression
}

\author{
KUN HAN, ZHAO JIAN LI and PENG SUN
}

\begin{abstract}
Department of Neurosurgery, Affiliated Hospital of Qingdao University, Qingdao, Shandong 266000, P.R. China
\end{abstract}
Received January 21, 2018; Accepted September 17, 2018

DOI: $10.3892 /$ or.2018.6823

\begin{abstract}
The aim of the present study was to analyze the possible association between microRNA-494 (miR-494) and cell proliferation in glioma cancer. Firstly, the expression of miR-494 was revealed to be upregulated in patients with glioma, compared with the normal group. Next, anti-miR-494 mimics were used to decrease the expression of miR-494 in glioma cancer cells, which subsequently induced apoptosis, and inhibited cell growth and migration. Downregulation of miR-494 expression induced phosphatase and tensin homolog (PTEN) and suppressed the protein kinase B/mechanistic target of rapamycin pathway (Akt/mTOR) pathway in glioma cancer cells. By contrast, overexpression of miR-494 by miR-494 mimics promoted cell growth and migration, and suppressed the apoptosis of glioma cancer via the Akt/mTOR pathway by PTEN expression. Furthermore, a PTEN inhibitor was used to attenuate the function of miR-494 in glioma cancer autophagy through Akt/mTOR pathway. The promotion of PTEN promoted the function of anti-miR-494 on glioma cancer cell growth through Akt/mTOR pathway. Collectively, these results demonstrate that the effect of miRNA-494 on the proliferation and migration glioma cancer cells was mediated through Akt/mTOR pathway by PTEN expression.
\end{abstract}

\section{Introduction}

Glioma cancer is also referred to as neuroectodermal tumor or neuroepithelial tumor since the tumor develops in the neuroderm. According to the World Health Organization, $\sim 75 \%$ malignant primary brain tumors are glioma cancer (1).

Correspondence to: Professor Peng Sun, Department of Neurosurgery, Affiliated Hospital of Qingdao University, 16 Jiangsu Road, Qingdao, Shandong 266000, P.R. China

E-mail: s5598701574@163.com

Key words: microRNA-494, glioma cancer, cell proliferation, protein kinase $\mathrm{B} /$ mechanistic target of rapamycin pathway, phosphatase and tensin homolog
Meanwhile, the annual morbidity rate of 5/10,000 and the high mortality rate of glioma have attracted extensive attention (2). It has been reported in the literature that the annual morbidity rate of glioma in China is 3-6/100,000, with a higher incidence among male patients than female patients. In addition, $\sim 30,000$ patients succumb to this disease annually (3). Glioma can occur at all age groups, which is dominated by adults, with 30-40 years being the peak age of morbidity (2). Glioma in adults is commonly observed in the supratentorial region, while that in children is mostly in the cerebellum (4). The majority of gliomas are tumors with invasive growth, which are associated with the possibility of recurrence following resection (4). Common clinical symptoms of glioma are associated with the tumor site and pathological type of glioma (5).

As one of the most common malignant primary intracranial tumors, glioma cancer is the central nervous system tumor with the poorest therapeutic effects at present (6). Medical professionals have performed a large number of studies on the molecular biology of glioma, including its association with microRNA (miRNA) (7). It has been demonstrated that microRNA is associated with glioma stem cells (7). This is of crucial importance to study microRNA and it has attracted extensive attention (8). miRNA acts on downstream target genes or proteins through multiple signaling pathways and transcription factors (8). Therefore, miRNAs can regulate the expression of oncogenes or tumor suppressor genes, and result in cell cycle changes in glioma stem cells. In addition, this will lead to changes in cell apoptosis, proliferation and differentiation, and affect the differentiation of glioma and its self-renewal ability (9). miRNA expression has been demonstrated to be upregulated or downregulated in glioma stem cells. This renders corresponding changes in signaling pathways, or all types of transcription or growth factors (9). Furthermore, certain tumor stem cell-related characteristics can also be affected, including proliferation, differentiation, invasion and migration. Therefore, microRNA can inhibit the growth and proliferation of tumor stem cells (9).

The phosphatase and tensin homolog (PTEN) gene is a new gene isolated from the homologous deletion region of primary breast cancer (10). It is the first tumor suppressor gene possessing dual phosphatase activity discovered thus far, and is located on chromosome 10q23 (10). The PTEN 
gene inhibits tumor cell proliferation, induces apoptosis, and suppresses tumor cell migration and local adhesion, thereby exerting effects on the tumor (10). Deletion or mutation of the PTEN gene can result in abnormal cell proliferation (11). Loss of homozygosity and heterozygosity, as well as abnormal promoter methylation of the gene, can result in its inactivation (11). A previous study demonstrated that the PTEN gene is associated with the genesis and development of multiple solid tumors (12). A previous study suggested that abnormal methylation of the PTEN gene promoter may be observed in different types of tumors (11), including breast cancer, esophageal cancer, thyroid carcinoma, renal carcinoma, oral squamous cell carcinoma and ovarian clear cell carcinoma (13).

The phosphoinositide 3-kinase (PI3K)/protein kinase B (Akt) signaling pathway is extensively distributed in cells. It exerts important physiological functions through multiple pathways, including regulating cell cycle and cellular energy metabolism (14). It has been demonstrated that the PI3K/Akt signaling pathway is excessively activated in glioma cancer, suggesting an association between this pathway, and the genesis and development of glioma (15). Meanwhile, PI3K and Akt may be effective targets for the clinical treatment of glioma cancer (15). Zhang et al (16) reported that miRNA-494 promotes colorectal (16), epithelial ovarian (17) and pancreatic cancer (18). The aim of the present study was to analyze the possible association between microRNA-494 and cell proliferation in glioma.

\section{Materials and methods}

Glioma samples. The peripheral blood of patients with glioma $(n=58$; age range, 44-70 years; mean age, $57 \pm 12.5$ years; 28 males and 30 females) and healthy volunteers $(n=28$; age range, 51-69 years; mean age, 59.5 \pm 9 years; 14 males and 14 females) were collected and centrifuged at $10,000 \times \mathrm{g}$ at $4^{\circ} \mathrm{C}$ for $10 \mathrm{~min}$. Serum was collected and stored at $-70^{\circ} \mathrm{C}$, prior to being used for quantitative polymerase chain reaction (qPCR).

RNA isolation and $q P C R$. Total RNA was extracted from serum samples using TRIzol reagent (Invitrogen; Thermo Fisher Scientific, Inc., Waltham, MA, USA), cDNA was amplified from $10 \mathrm{ng}$ RNA using Taqman MicroRNA assays (Applied Biosystems; Thermo Fisher Scientific, Inc.). cDNA was prepared using SYBR Premix Ex Taq ${ }^{\mathrm{TM}}$ II (Takara Biotechnology Co., Ltd., Dalian, China). MicroRNA-494 forward, 5'-CATAGCCCGTGAAACATACACG-3' and reverse, 5'-GTGCAGGGTCCGAGGT-3'; and U6 forward, 5'-CGCTTCGGCAGCACATATACTA-3' and reverse, 5'-GCG AGCACAGAATTAATACGAC-3'. The thermocycling conditions for this stage were as follows: $95^{\circ} \mathrm{C}$ for $5 \mathrm{~min}$; 40 cycles of denaturation at $95^{\circ} \mathrm{C}$ for $25 \mathrm{sec}$; and annealing/extension at $60^{\circ} \mathrm{C}$ for $30 \mathrm{sec}$. The miRNA expression was determined using the comparative $\mathrm{Cq}\left(2^{-\Delta \Delta \mathrm{Cq}}\right)$ method method (19). Low miRNA-494 expression was categorized as 1-2 times that of the control group (U6), and high miRNA-494 expression was categorized as $>2$ times that of the control group (U6).

Cell lines and transfection. Human glioma U251 cells were maintained in RPMI-1640 medium $\left(\right.$ HyClone $^{\mathrm{TM}}$; GE Healthcare Life Sciences, Logan, UT, USA), supplemented with $10 \%$ fetal bovine serum (Gibco; Thermo Fisher Scientific, Inc.) and cultured at $37^{\circ} \mathrm{C}$ in a $5 \% \mathrm{CO}_{2}$ incubator. A total of $100 \mathrm{ng}$ anti-miR-494, miR-494, si-PTEN, PTEN plasmid and negative control were synthesized by Guangzhou RiboBio Co., Ltd. (Guangzhou, China). Anti-miR-494 (5'-UUCUCC GAACGUGUCACGUUU-3' and 5'-ACGUACACGUUC GGAGAAUU-3'), miR-494 (5'UGAAACAUACACGGG AAACCUC3' and 5'-GGUUUCCCGUGUAUGUUUCAU U-3') and negative control were transfected into cells using Lipofectamine 2000 (Invitrogen; Thermo Fisher Scientific, Inc.). Next, anti-miR-494 and si-PTEN (cat. no. sc-44272; Santa Cruz Biotechnology) or miR-494 and PTEN plasmid (5'-ACTCTTGCCTGGTTGCAAGTGTCAA-3' and 5'-TCT GAATTT TTT TTTATCAAGAGGGGATAA A-3') were transfected into cells using Lipofectamine 2000. After $4 \mathrm{~h}$ of transfection, fresh RPMI-1640 medium was added to cells.

Luciferase reporter assay. The PTEN 3'-UTR containing the microRNA-494 target site was cloned into the pGL3 luciferase reporter vector (Sangon Biotech Co., Ltd. Shanghai, China). The PTEN 3'-UTR containing the microRNA-494 plasmid was transfected into cells using Lipofectamine 2000. A Dual-Luciferase Reporter assay system (Promega Corporation, Madison, WI, USA) was used to measure luciferase activity levels at $48 \mathrm{~h}$ after transfection through comparison with Renilla luciferase activity using a Renilla luciferase activity kit (Promega Corporation).

MTT assay. Cell proliferation was analyzed at 24, 48 and $72 \mathrm{~h}$ after transfection by MTT assay. A total of $20 \mu \mathrm{l} \mathrm{MTT}$ was added to cells, followed by incubation for $4 \mathrm{~h}$ at $37^{\circ} \mathrm{C}$. Medium was removed and $150 \mu \mathrm{l}$ dimethyl sulfoxide (DMSO) was added to cells, followed by agitation for $20 \mathrm{~min}$ at $37^{\circ} \mathrm{C}$. Optical absorbance was read by EnSpire Multimode Plate Reader (PerkinElmer, Inc., Waltham, MA, USA) at $490 \mathrm{~nm}$.

Transwell assay for cell invasion. Cells $\left(1 \times 10^{5}\right)$ were seeded into upper chambers (8- $\mu$ m pore size; Merck KGaA, Darmstadt, Germany) in RPMI-1640 medium, and RPMI-1640 medium supplemented with $10 \%$ fetal bovine serum was plated into the lower chambers. Meanwhile, cells were placed into the upper chambers and pre-coated with Matrigel (BD Biosciences, Franklin Lakes, NJ, USA). Following incubation for $48 \mathrm{~h}$, the chambers were fixed with $4 \%$ paraformaldehyde at room temperature for $30 \mathrm{~min}$ and then stained with $0.1 \%$ crystal violet (BD Biosciences) at room temperature for $15 \mathrm{~min}$. Cell was observed using a Zeiss Axioplan 2 confocal microscope (magnification, x100; Carl Zeiss MicroImaging).

Flow cytometric analysis and caspase-3/-9 activity. Cell proliferation was analyzed at $48 \mathrm{~h}$ after transfection, and cells were resuspended in PBS and stained with Annexin V-fluorescein isothiocyanate/propidium iodide (5 $\mu \mathrm{l}$; BD Biosciences) for $15 \mathrm{~min}$ at room temperature. The stained cells were quantified using a flow cytometer (BD Influx ${ }^{\mathrm{TM}}$ Instrument; BD Biosciences) and analyzed using FlowJo 7.6.1 software (FlowJo LLC, Ashland, OR, USA) Protein was extracted from cells using radioimmunoprecipitation assay (RIPA) buffer and the liquid was placed in an ice bath for $30 \mathrm{~min}$. Total protein concentration was determined using a bicinchoninic 
A
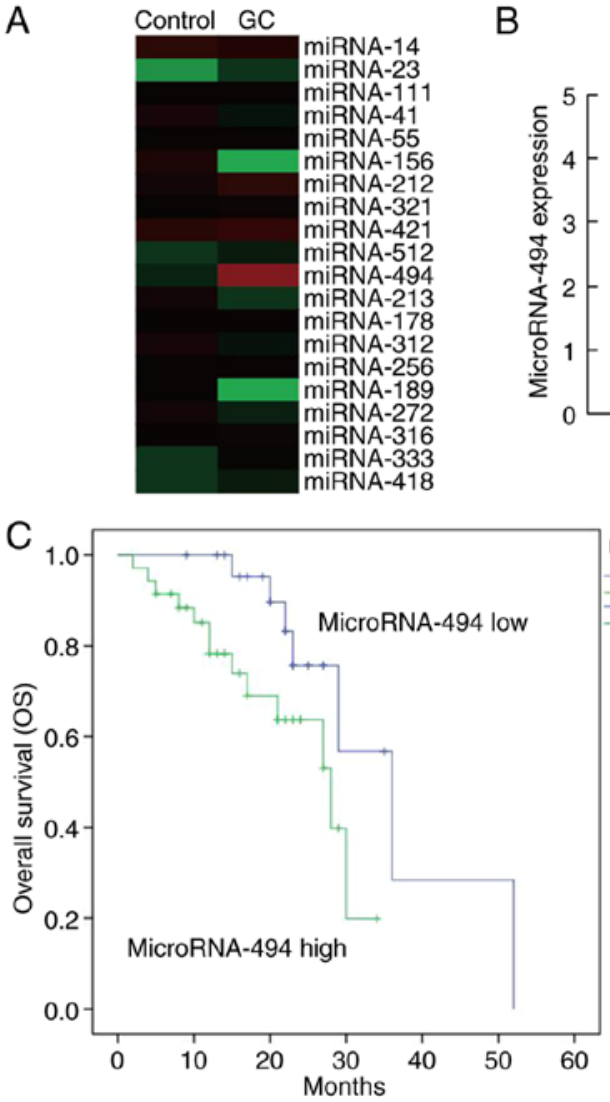

B

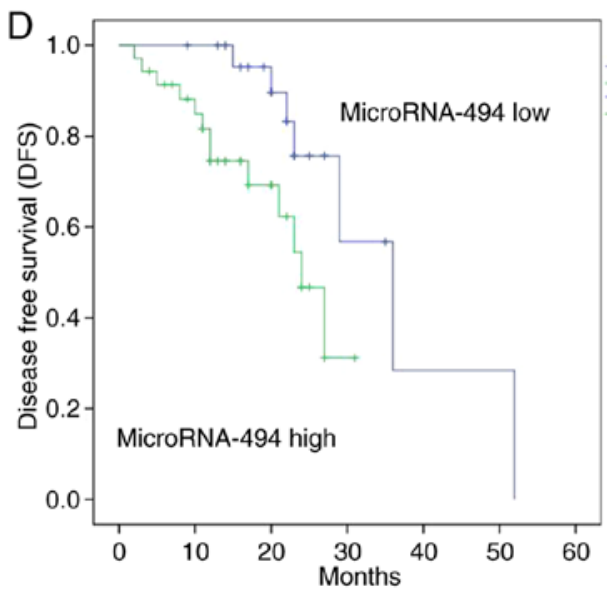

Figure 1. miRNA-494 expression. (A) Gene chip for miRNA-494 expression. (B) Quantitative polymerase chain reaction for miRNA-494 expression. (C) Overall survival and (D) disease-free survival of patients with glioma. Control, healthy volunteer group ( $\mathrm{n}=28$ ); GC, glioma cancer ( $\mathrm{n}=58$ ); miRNA, microRNA. ${ }^{\# \#} \mathrm{P}<0.01$, compared with the healthy volunteer group.

acid (BCA) protein assay kit. Proteins were used to measure caspase-3/-9 activity, and optical absorbance was read by an EnSpire Multimode Plate Reader (PerkinElmer, Inc.) at $405 \mathrm{~nm}$.

Western blot analysis. Protein was extracted from cells using RIPA buffer and the liquid was placed in an ice bath for $30 \mathrm{~min}$. Total protein concentration was determined using BCA protein assay kit. Proteins were resolved through 8-10\% SDS-PAGE, prior to transfer onto PVDF membranes (EMD Millipore, Billerica, MA, USA). The membranes were blocked with $5 \%$ dry skimmed milk for $1 \mathrm{~h}$ at $37^{\circ} \mathrm{C}$, and probed with Bax (cat. no. ab32503; 1:2,000; Abcam, Cambridge, MA, USA), LC3 (cat. no. ab48394; 1:2,000; Abcam), PTEN (cat. no. ab228466, 1:2,000; Abcam), p-Akt (cat. no. 4060; 1:2,000; Cell Signaling Technology, Inc.), p-mTOR (cat. no. 5536; 1:2,000; Cell Signaling Technology, Inc.) and GAPDH (cat. no. ab8245, 1:5,000; Abcam) primary antibodies at $4^{\circ} \mathrm{C}$ for 6-8 h. Membranes were washed with TBST, incubated with horseradish peroxidase-conjugated goat anti-rabbit IgG antibodies (cat. no. 7074; 1:5,000; Cell Signaling Technology, Inc.) at room temperature for $2 \mathrm{~h}$, visualized using chemiluminescence (Pierce; Thermo Fisher Scientific, Inc.) and analyzed using Image_Lab_3.0 software (Bio-Rad Laboratories, Inc., Hercules, CA, USA).

Statistical analysis. The results are expressed as the mean \pm standard error of the mean. Data were analyzed using
Student's t-test or one-way analysis of variance, followed by Tukey's post hoc test. $\mathrm{P}<0.05$ was considered to indicate a statistically significant difference.

\section{Results}

The expression of $m i R-494$. To determine whether miR-494 from the serum of glioma samples, the miR-494 expression was examined. As demonstrated in Fig. 1A and B, miR-494 expression was upregulated in the serum of patients with glioma, compared with the normal group. Subsequently, the association between miR-494 expression and survival rate was analyzed in patients with glioma. As a result, the overall survival (OS) and disease-free survival (DFS) rates in patients with glioma with a lower expression of miR-494 were higher than those in patients with a higher expression of miR-494 (Fig. 1C and D).

Anti-miR-494 inhibits the growth and migration of glioma cells. Furthermore, the growth and migration of glioma cells was assessed following downregulation of miR-494. As demonstrated in Fig. 2A-D, anti-miR-494 mimics decreased miR-494 expression, and inhibited the growth and migration of glioma cells, compared with the control group. Furthermore, caspase-3 and caspase-9 activity levels, apoptosis rate and Bax protein expression were induced by anti-miR-494 in glioma, compared with the control group (Fig. 2E-H). 


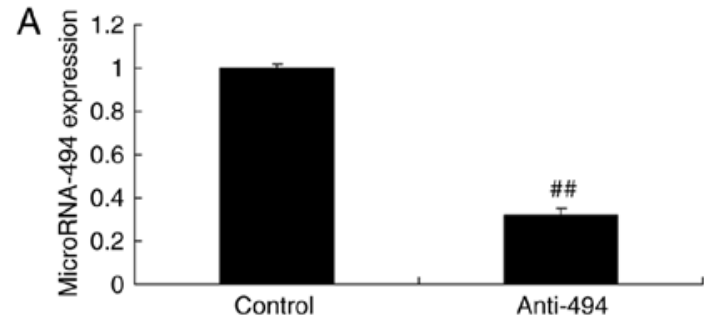

C

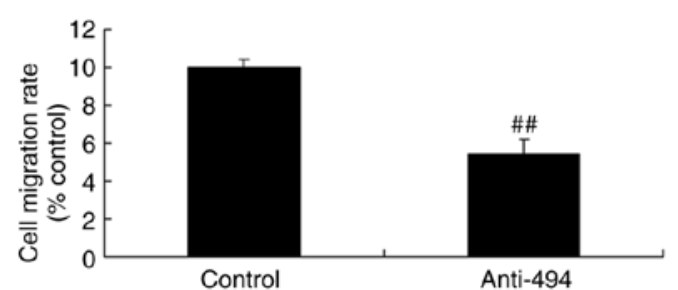

$\mathrm{E}$

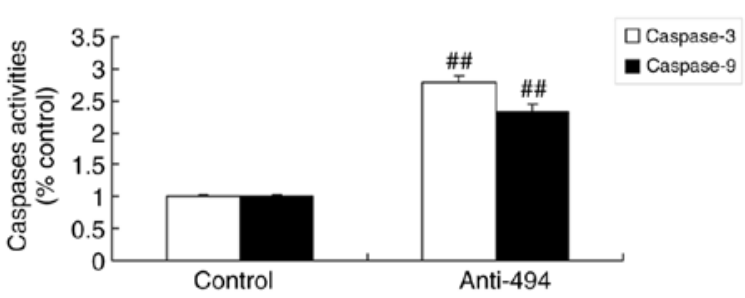

G

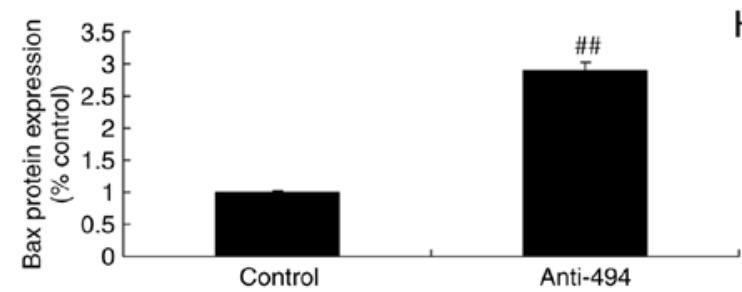

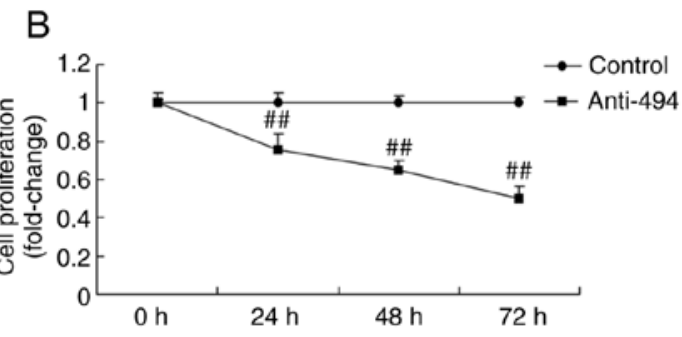

$\mathrm{D}$

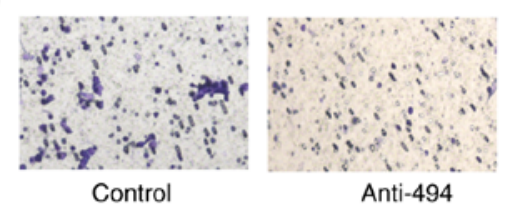

$\mathrm{F}$

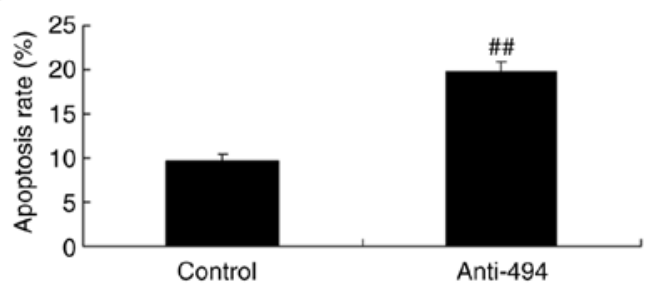

$\mathrm{H}$

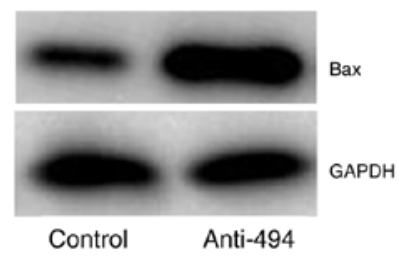

Figure 2. Anti-microRNA-494 inhibited the growth and migration of glioma cells. (A) Expression of microRNA-494, (B) cell proliferation, (C) migration rate and (D) migration rate by statistical analysis, (E) Caspase-3/-9 activity, (F) apoptosis rate, and Bax protein expression by (G) statistical analysis and (H) western blot analysis in glioma. Control, negative control group $(\mathrm{n}=3)$; anti-miRNA-494, downregulation of microRNA-494 $(\mathrm{n}=3)$. ${ }^{\# \#} \mathrm{P}<0.01$, compared with the negative control group.

miR-494 promotes the growth and migration of glioma cells. The expression of miR-494 was upregulated by miR-494 mimics, followed by analysis of the function of miR-494 on the growth and migration of glioma cells. miR-494 expression of glioma in miR-494 group was increased, compared with the control group (Fig. 3A). Growth and migration of glioma cells were promoted by overexpression of miR-494, compared with the control group (Fig. 3B-D). However, caspase-3 and caspase- 9 activity levels, apoptosis rate and Bax protein expression in glioma were suppressed by miR-494 upregulation, compared with the control group (Fig. 3E-H).

The effects of miR-494 regulates the PTEN/Akt/mTOR pathway in glioma. As demonstrated in Fig. 4A, PTEN is a direct target of miR-494. Immunofluorescence revealed that anti-miR-494 induced the protein expression of PTEN in glioma, compared with the control group (Fig. 4B). Next, western blot analysis was used to measure changes in the PTEN/Akt/mTOR pathway in glioma using anti-miR-494 or miR-494 mimics. As a result, PTEN protein expression was induced, and p-Akt and p-mTOR protein expression were suppressed by anti-miR-494 in glioma, compared with the control group (Fig. 5A-D). Overexpression of miR-494 suppressed PTEN protein expression, and induced p-Akt and p-mTOR protein expression in glioma, compared with the control group (Fig. 5E-H).

The inhibition of PTEN reduces the function of miR-494 in glioma cell growth through the Akt/mTOR pathway. To determine the role of PTEN in the function of the miR-494/Akt/mTOR pathway in glioma, si-PTEN was used to suppress PTEN protein expression in glioma following anti-miR-494 transfection, compared with the anti-miR-494 alone group (Fig. 6A and B). Consequently, the protein expression of $\mathrm{p}$-Akt and $\mathrm{p}$-mTOR in glioma was induced by the inhibition of PTEN, compared with anti-miR-494 (Fig. 6A, C and D). The function of miR-494 in the inhibition of cell growth and migration in glioma was reversed following PTEN interference, compared with anti-miR-494 alone (Fig. 7A-C). The inhibition of PTEN reduced the effect of miR-494 on glioma cell apoptosis and Bax protein expression, compared with anti-miR-494 (Fig. 7D-G).

The promotion of PTEN promotes the function of miR-494 in glioma cancer cell proliferation through the Akt/mTOR pathway. Finally, to further determine the role of PTEN 
A

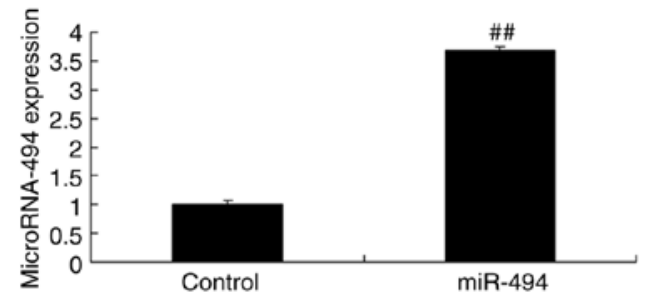

C

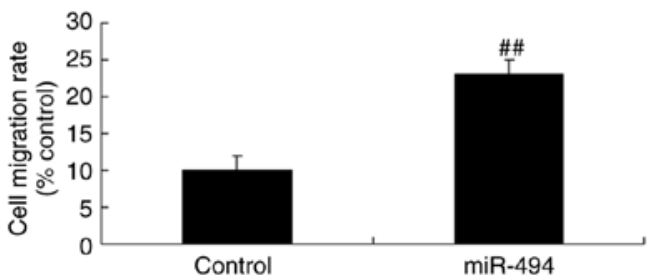

$\mathrm{E}$

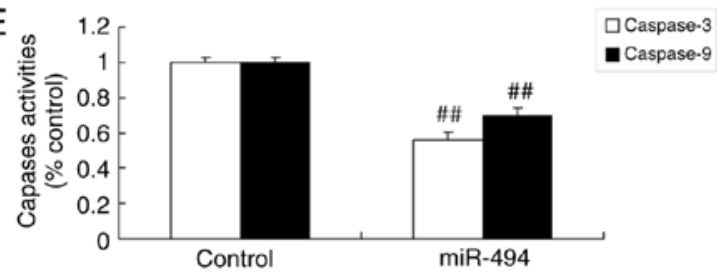

G

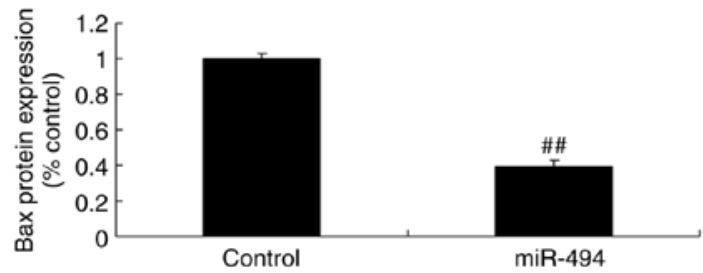

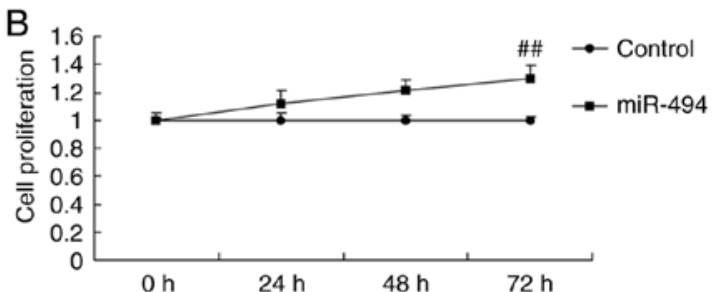

D

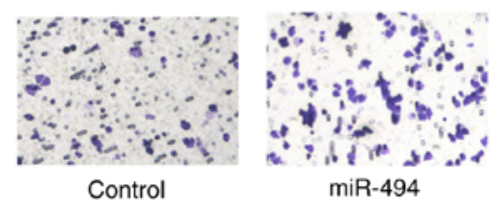

$\mathrm{F}$

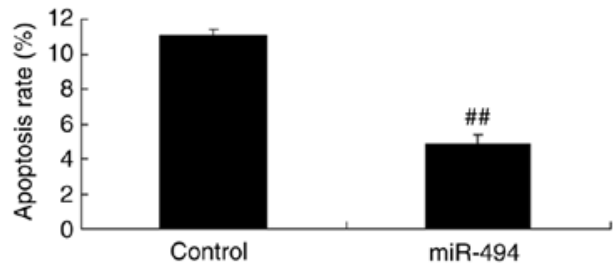

$\mathrm{H}$



Figure 3. MicroRNA-494 promoted the growth and migration of glioma cells. (A) Expression of microRNA-494, (B) cell proliferation, (C) migration rate and (D) migration rate by statistical analysis, (E) Caspase-3/-9 activity, (F) apoptosis rate, and Bax protein expression by (G) statistical analysis and (H) western blot analysis in glioma. Control, negative control group $(\mathrm{n}=3)$; miRNA-494, overexpression of microRNA-494 $(\mathrm{n}=3)$. ${ }^{\#} \mathrm{P}<0.01$, compared with the negative control group; miR, microRNA.
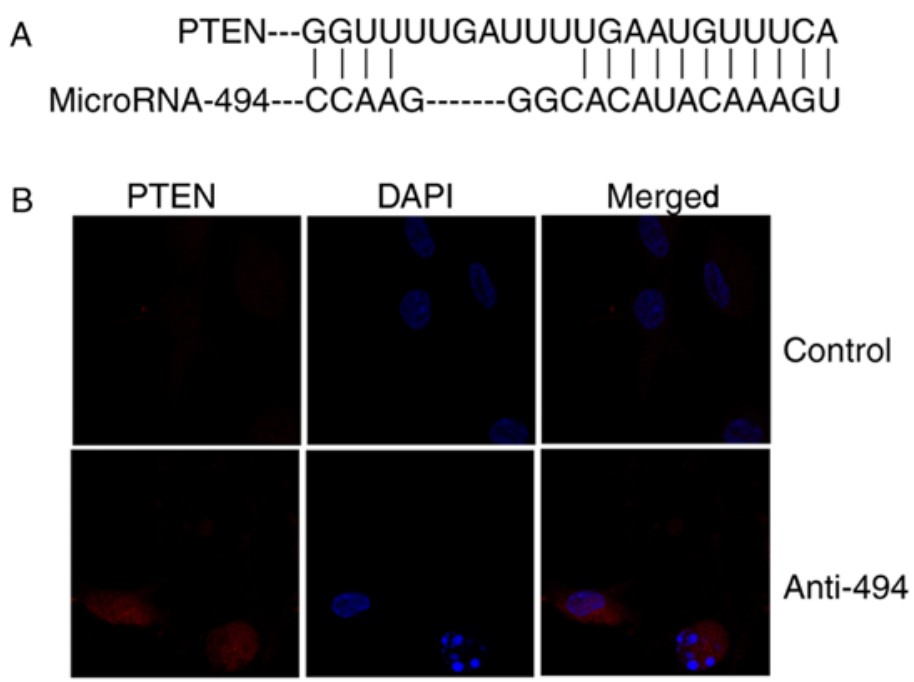

Figure 4. Effects of the microRNA-494-regulated PTEN pathway in glioma. (A) PTEN is a direct target of microRNA-494. (B) Immunofluorescence revealed PTEN protein expression. Control, negative control group $(\mathrm{n}=3)$; anti-miRNA-494, downregulation of microRNA-494 ( $\mathrm{n}=3$ ). PTEN, phosphatase and tensin homolog.

in the anticancer effect of miR-494 on glioma, PTEN plasmid induced the protein expression in glioma following anti-miR-494, compared with anti-microRNA-494 alone (Fig. 8A and B). The promotion of PTEN suppressed the protein expression of $\mathrm{p}$-Akt and p-mTOR in glioma following
anti-miR-494 transfection, compared with anti-miR-494 alone (Fig. 8A, C and D). The promotion of PTEN reduced cell growth and migration, and induced apoptosis and Bax protein expression in glioma following anti-miR-494, compared with anti-miR-494 alone (Fig. 9). 
A

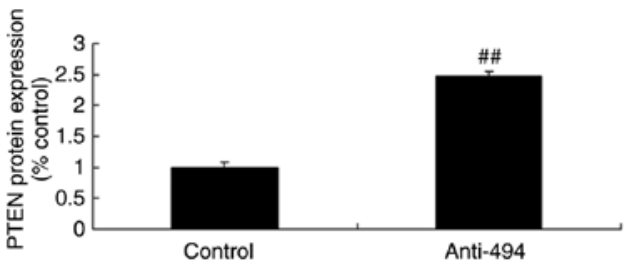

C

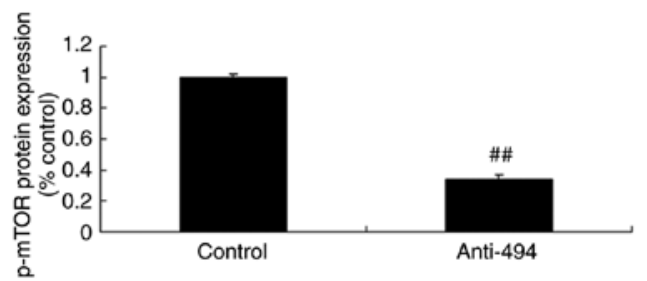

E

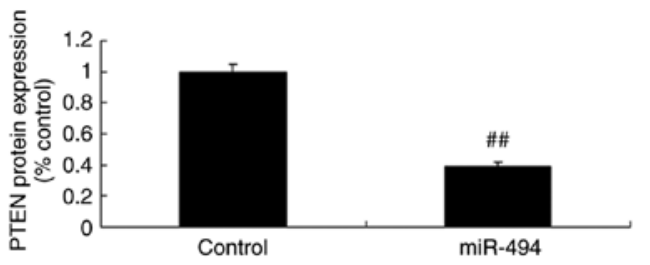

G

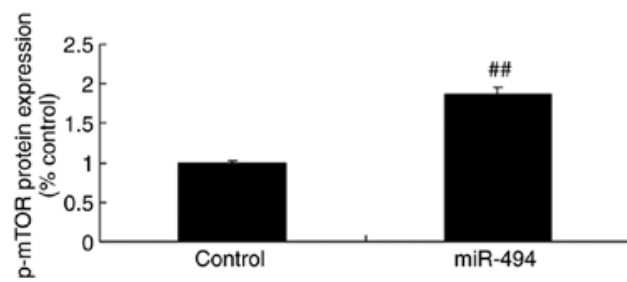

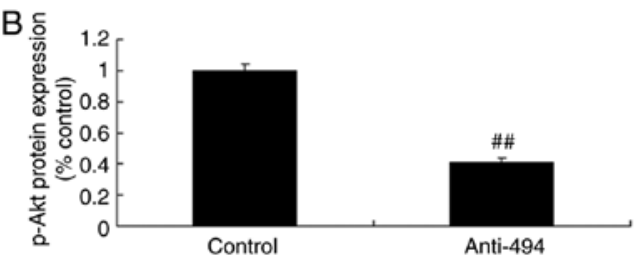

D

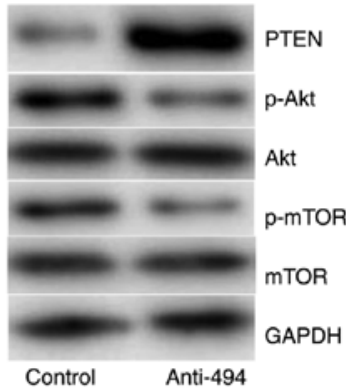

$\mathrm{F}$

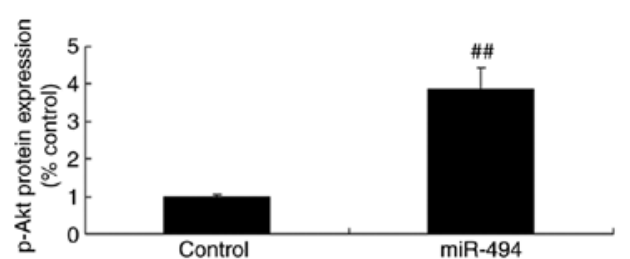

$\mathrm{H}$

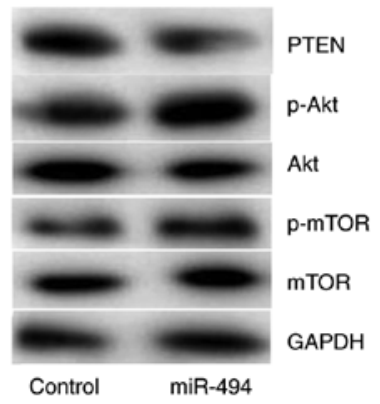

Figure 5. Effects of the microRNA-494-regulated PTEN/Akt/mTOR pathway in glioma. (A) PTEN, (B) p-Akt and (C) p-mTOR protein expression by statistical analysis, and (D) western blot analysis following downregulation of miRNA-494. (E) PTEN, (F) p-Akt and (G) p-mTOR protein expression by statistical analysis, and $(\mathrm{H})$ western blot analysis following overexpression of miRNA-494. Control, negative control group (n=3); anti-miRNA-494, downregulation of miRNA-494 ( $n=3)$; miRNA-494, overexpression of miRNA-494 $(n=3) .{ }^{* \#} \mathrm{P}<0.01$, compared with the negative control group. PTEN, phosphatase and tensin homolog; Akt, protein kinase B; mTOR, mechanistic target of rapamycin; p-, phosphorylated; miR, microRNA.
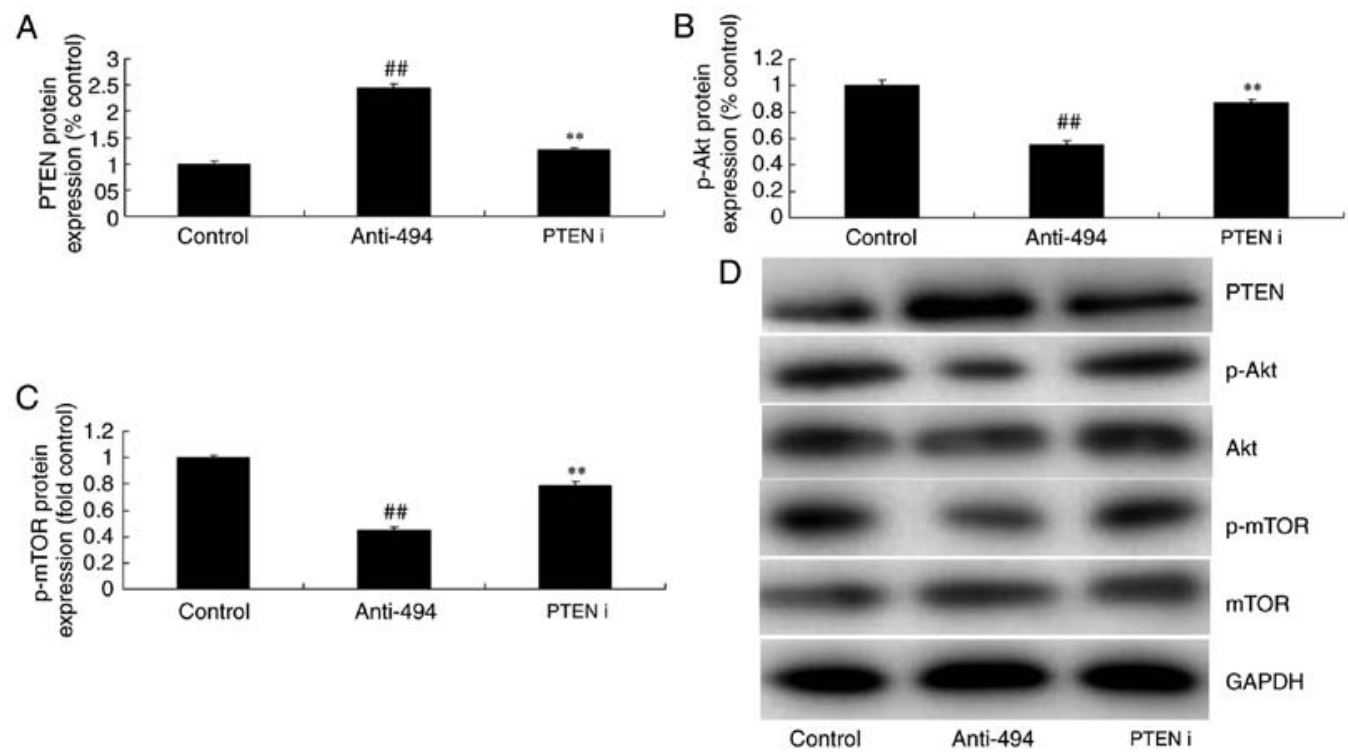

Figure 6. Inhibition of PTEN reduces the effect of microRNA-494 on the Akt/mTOR pathway. (A) PTEN, (B) p-Akt and (C) p-mTOR protein expression by statistical analysis, and (D) western blot analysis. Control, negative control group $(n=3)$; anti-miRNA-494, downregulation of microRNA-494 (n=3); PTEN i, si-PTEN and downregulation of microRNA-494 $(\mathrm{n}=3) .{ }^{\# \#} \mathrm{P}<0.01$, compared with the negative control group; ${ }^{* *} \mathrm{P}<0.01$, compared with the downregulation of microRNA-494 group. PTEN, phosphatase and tensin homolog; Akt, protein kinase B; mTOR, mechanistic target of rapamycin; p-, phosphorylated; miR, microRNA; I, interference. 
A

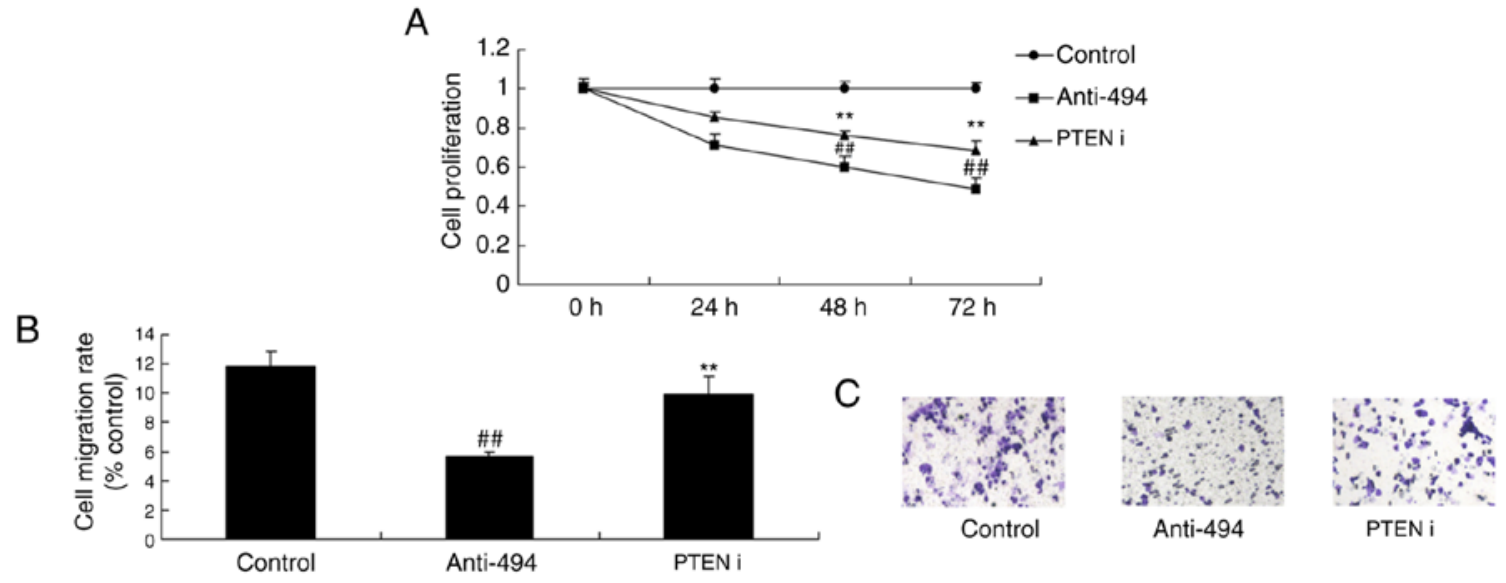

D

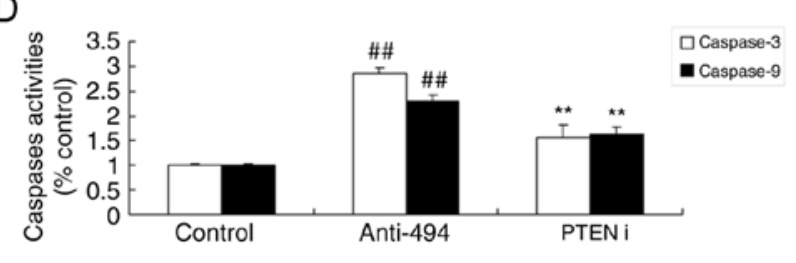

$\mathrm{F}$

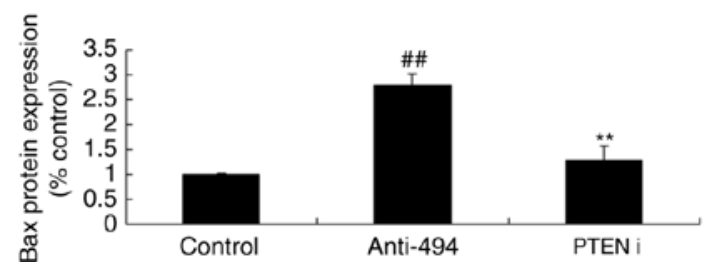

E

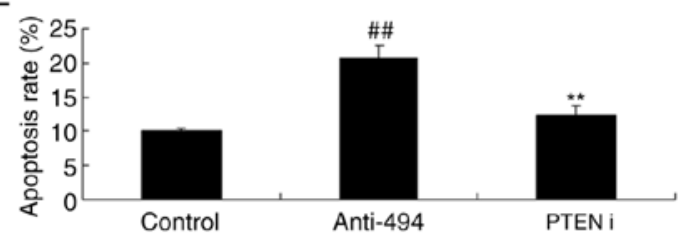

G

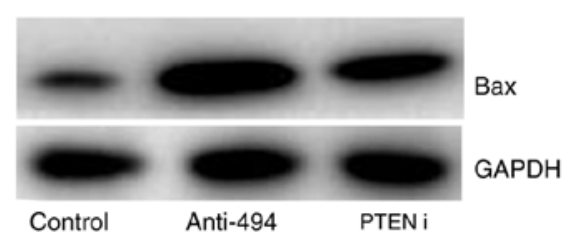

Figure 7. Inhibition of PTEN reduces the effect of microRNA-494 on glioma cancer cell growth through the Akt/mTOR pathway. (A) Cell proliferation, (B) migration rate and (C) migration rate by statistical analysis, (D) Caspase-3/-9 activity, (E) apoptosis rate and Bax protein expression by (F) statistical analysis and (G) western blot analysis in glioma. Control, negative control group $(n=3)$; anti-miRNA-494, downregulation of microRNA-494 ( $\mathrm{n}=3$ ); PTEN i, si-PTEN and downregulation of microRNA-494 (n=3). ${ }^{\# \#} \mathrm{P}<0.01$, compared with the negative control group; ${ }^{* * *} \mathrm{P}<0.01$, compared with the downregulation of microRNA-494 group. PTEN, phosphatase and tensin homolog; Akt, protein kinase B; mTOR, mechanistic target of rapamycin; i, interference.

A

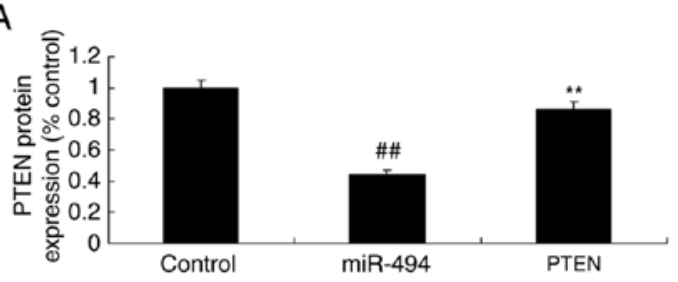

C

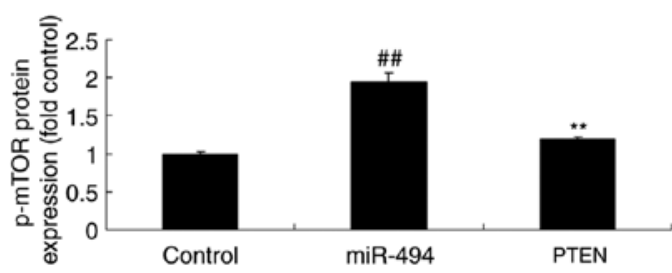

B

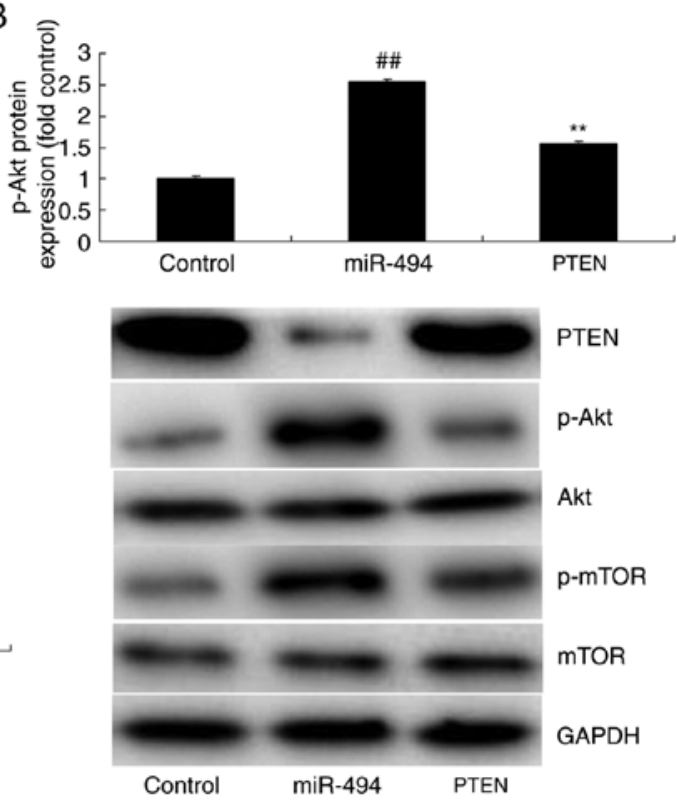

Figure 8. Promotion of PTEN promotes the effect of microRNA-494 on the Akt/mTOR pathway. (A) PTEN, (B) p-Akt and (C) p-mTOR protein expression by statistical analysis, and (D) western blot analysis. Control, negative control group ( $\mathrm{n}=3$ ); miR-494, overexpression of miR-494 ( $\mathrm{n}=3$ ); miR-494 + PTEN, PTEN plasmid overexpression of miR-494 $(\mathrm{n}=3) .{ }^{\#} \mathrm{P}<0.01$, compared with the negative control group ${ }^{* *} \mathrm{P}<0.01$, compared with the overexpression of microRNA-494 group. PTEN, phosphatase and tensin homolog; Akt, protein kinase B; mTOR, mechanistic target of rapamycin; p-, phosphorylated; i, interference; miR, microRNA. 


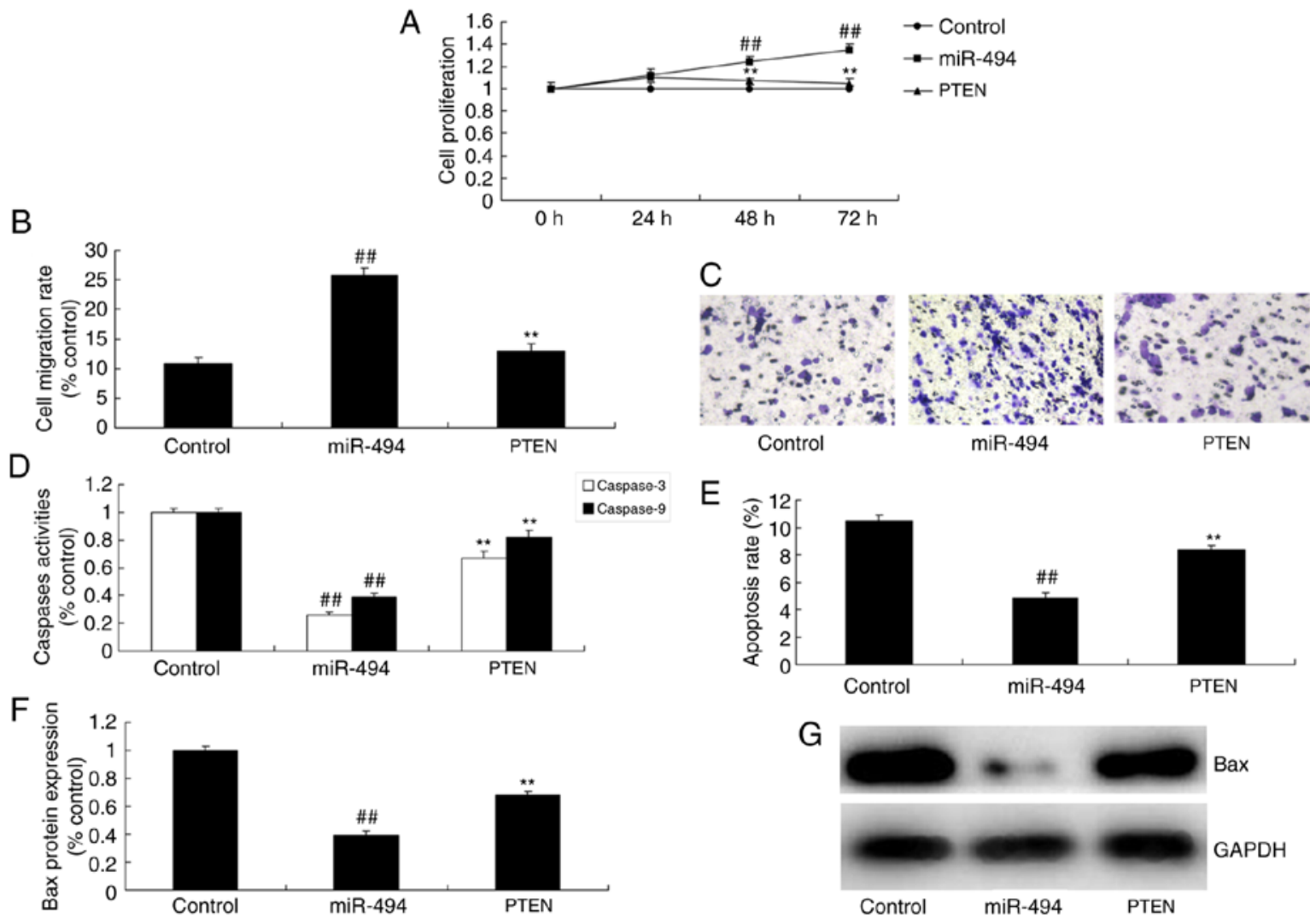

Figure 9. Promotion of PTEN promotes the effect of microRNA-494 on glioma cancer cell growth through the Akt/mTOR pathway. (A) Cell proliferation, (B) migration rate and (C) migration rate by statistical analysis, (D) Caspase-3/-9 activity, (E) apoptosis rate, and Bax protein expression by (F) statistical analysis and (G) Bax protein expression of glioma. Control, control negative group ( $\mathrm{n}=3)$; miRNA-494, overexpression of miR-494 (n=3); miR-494 + PTEN, PTEN plasmid overexpression of miR-494 $(n=3)$. ${ }^{\# \#} \mathrm{P}<0.01$, compared with the negative control group; ${ }^{* *} \mathrm{P}<0.01$, compared with overexpression of miR-494 group. PTEN, phosphatase and tensin homolog; Akt, protein kinase B; mTOR, mechanistic target of rapamycin; miR, microRNA.

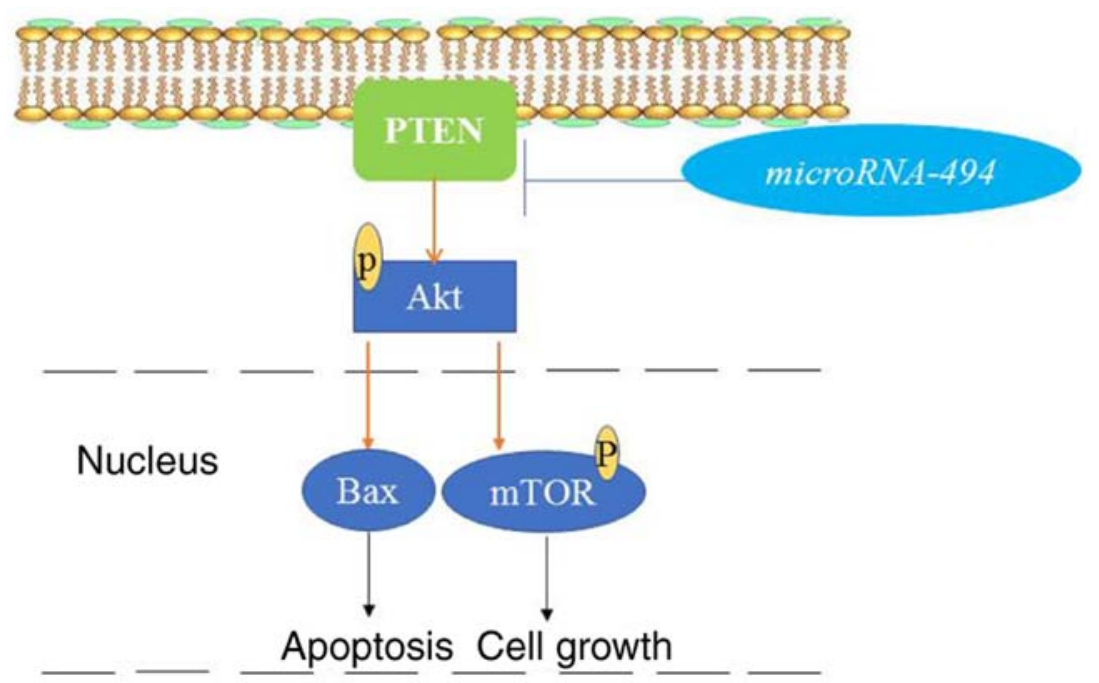

Figure 10. Effect of microRNA-494 promotes glioma cell proliferation and migration through the Akt/mTOR pathway by PTEN expression. Akt, protein kinase B; mTOR, mechanistic target of rapamycin; PTEN, phosphatase and tensin homolog.

\section{Discussion}

Glioma is an intracranial tumor with a high morbidity rate and it cannot be cured (4). WHO has classified it as the most severe astrocytoma with the highest degree of invasion (20). Previous studies have demonstrated that miRNA expression profiles in glioma and multiple malignant tumor tissues are different from those in normal tissues $(4,21)$. These differentially expressed microRNAs exert irreplaceable functions in the genesis and development of tumors through regulating the expression of oncogenes and tumor suppressor genes (20). Therefore, studying microRNA is required in order to elucidate the pathogenesis, diagnosis and treatment of cancer (22). Furthermore, the present study demonstrated 
that microRNA-494 expression is upregulated in the serum of patients with glioma, compared with the normal group. Taken together, the results of the present study suggested that miR494 acts as an oncogene in glioma. Zhang et al (16) reported that microRNA-494 promotes colorectal, epithelial ovarian (17) and pancreatic cancer (18). These results are in line with those of the present study and demonstrated that miR-494 participates in the development and progression of glioma.

In the pathogenesis of autophagy, the most dominant morphological feature of autophagy is the presence of a large amount of foam-like structures in cells (13). They are the double membrane phagocytic vacuoles that contain cytoplasm and organelles. The genesis of autophagy can be classified into the following 4 steps based on this (23): Autophagy induction, formation of autophagy vacuoles, fusion of autophagy vacuoles with lysosome, and degradation and recycling of materials. There are numerous cellular transduction signals regulating autophagy. Of them, PI3K and mTOR are relatively well known (24). PI3K is of crucial importance in the formation of early phagocytic vacuoles. For instance, PIK3C3-BECN1 in mammals serves a key role in regulating phosphatidylinositol in autophagy. In addition, it serves an important role in the formation of double molecular membrane structure of autophagy (25). The results of the present study demonstrated that anti-microRNA-494 induced PTEN expression and suppressed the Akt/mTOR pathway in glioma. Su et al (25) demonstrated that miR-494 upregulates the PI3K/Akt pathway through targeting PTEN in ischemia/reperfusion injury (26). Li et al (26) demonstrated that miR-494-3p regulates cellular proliferation and apoptosis by PTEN/Akt signaling in human glioblastoma cells (27). These results demonstrated that miR-494 participated in the growth and apoptosis of human glioblastoma cells, and it was established that PTEN is a direct target of miR-494 and that miR-494 regulates the Akt/mTOR pathway through PTEN expression.

The PTEN gene can promote autophagy. As a type of lipid phosphatase, PTEN can transform its substrate 3,4,5-phosphatidylinostiol triphosphate into 4,5-diphosphoinositide (28). Therefore, it serves a negative regulatory role in the PI3K-Akt signal transduction pathway. This pathway involves multiple downstream events, including apoptosis inhibition and stimulation of protein synthesis through the sirolimus target protein (mTOR) pathway (11). In the present study, the inhibition of PTEN reduced the microRNA-494-promoted apoptosis of glioma cells. Zhu et al (28) demonstrated that microRNA-494 inhibits apoptosis by modulating the PTEN/Akt/mTOR pathway in rats following spinal cord injury (29). However, only si-PTEN was used, and PTEN affected the function of miR-494, which demonstrated that PTEN only participated in the function of miR-494 in glioma. Further experimental methods are required in order to verify these results.

The present study demonstrated that microRNA-494 was involved in the growth and migration of glioma cells, and promoting the apoptosis of U251 cells. These results suggested that a high microRNA-494 expression level is a potential risk factor for glioma, and that the therapeutic potential of anti-miRNA-494 promoted the apoptosis of glioma cells through the Akt/mTOR pathway via PTEN expression (Fig. 10). Therefore, miRNA-494 may be considered as a potential therapeutic target for the treatment of glioma.

\section{Acknowledgements}

Not applicable.

\section{Funding}

No funding was received.

\section{Availability of data and materials}

The datasets generated and/or analyzed during the study are available from the corresponding author on reasonable request.

\section{Authors' contributions}

PS designed the experiment, analyzed the data and wrote the manuscript; $\mathrm{KH}$ and $\mathrm{ZJL}$ performed the experiment. All authors read and approved the final manuscript.

\section{Ethics approval and consent to participate}

This study was approved by the Ethics Committee of The First Affiliated Hospital of Qiingdao University.

\section{Patient consent for publication}

Not applicable.

\section{Competing interests}

The authors declare that they have no competing interests.

\section{References}

1. Buckner JC, Shaw EG, Pugh SL, Chakravarti A, Gilbert MR, Barger GR, Coons S, Ricci P, Bullard D, Brown PD, et al: Radiation plus procarbazine, CCNU, and vincristine in low-grade glioma. N Engl J Med 374: 1344-1355, 2016.

2. Crane CA, Han SJ, Ahn B, Oehlke J, Kivett V, Fedoroff A, Butowski N, Chang SM, Clarke J, Berger MS, et al: Individual patient-specific immunity against high-grade glioma after vaccination with autologous tumor derived peptides bound to the 96 KD chaperone protein. Clin Cancer Res 19: 205-214, 2013.

3. Pan E, Yu D, Yue B, Potthast L, Chowdhary S, Smith P and Chamberlain M: A prospective phase II single-institution trial of sunitinib for recurrent malignant glioma. J Neurooncol 110: 111-118, 2012.

4. Li HL, Cui XL, Zhang JN and Lin S: Chemotherapy alleviates subacute recurrent glioma-associated refractory cerebral edema by downregulating vascular endothelial growth factor. Med Oncol 31: 13, 2014.

5. Luo $X$, Zheng $X$ and Huang H: Protective effects of dexmedetomidine on brain function of glioma patients undergoing craniotomy resection and its underlying mechanism. Clin Neurol Neurosurg 146: 105-108, 2016.

6. Li P, Wang X, Shan Q, Wu Y and Wang Z: MicroRNA-130b promotes cell migration and invasion by inhibiting peroxisome proliferator-activated receptor-gamma in human glioma. Oncol Lett 13: 2615-2622, 2017.

7. Liu X, Chen J and Zhang J: AdipoR1-mediated miR-3908 inhibits glioblastoma tumorigenicity through downregulation of STAT2 associated with the AMPK/SIRT1 pathway. Oncol Rep 37: 3387-3396, 2017.

8. Pal R and Greene S: microRNA-10b is overexpressed and critical for cell survival and proliferation in medulloblastoma. PLoS One 10: $\mathrm{e} 0137845,2015$. 
9. Mazurowski MA, Clark K, Czarnek NM, Shamsesfandabadi P, Peters KB and Saha A: Radiogenomics of lower-grade glioma: Algorithmically-assessed tumor shape is associated with tumor genomic subtypes and patient outcomes in a multi-institutional study with The Cancer Genome Atlas data. J Neurooncol 133 27-35, 2017.

10. Wang MH, Lin CL, Zhang JJ, Weng ZP, Hu T, Xie Q and Zhong XY: Role of PTEN in cholera toxin-induced SWO38 glioma cell differentiation. Mol Med Rep 7: 1912-1918, 2013.

11. Elhag R, Mazzio EA and Soliman KF: The effect of silibinin in enhancing toxicity of temozolomide and etoposide in p53 and PTEN-mutated resistant glioma cell lines. Anticancer Res 35: 1263-1269, 2015

12. Lester A, Rapkins R, Nixdorf S, Khasraw M and McDonald K: Combining PARP inhibitors with radiation therapy for the treatment of glioblastoma: Is PTEN predictive of response? Clin Transl Oncol 19: 273-278, 2017.

13. Koul D, Wang S, Wu S, Saito N, Zheng S, Gao F, Kaul I, Setoguchi M, Nakayama K, Koyama K, et al: Preclinical therapeutic efficacy of a novel blood-brain barrier-penetrant dual $\mathrm{PI} 3 \mathrm{~K} / \mathrm{mTOR}$ inhibitor with preferential response in PI3K/PTEN mutant glioma. Oncotarget 8: 21741-21753, 2017.

14. Koul D, Shen R, Kim YW, Kondo Y, Lu Y, Bankson J, Ronen SM, Kirkpatrick DL,Powis G and Yung WK: Cellular and in vivo activity of a novel PI3K inhibitor, PX-866, against human glioblastoma. Neuro Oncol 12: 559-569, 2010.

15. Paul-Samojedny M, Pudelko A, Kowalczyk M, Fila-Daniłow A, Suchanek-Raif R, Borkowska P and Kowalski J: Combination therapy with AKT3 and PI3KCA siRNA enhances the antitumor effect of temozolomide and carmustine in T98G glioblastoma multiforme cells. BioDrugs 30: 129-144, 2016.

16. Zhang Y, Guo L, Li Y, Feng GH, Teng F, Li W and Zhou Q: MicroRNA-494 promotes cancer progression and targets adenomatous polyposis coli in colorectal cancer. Mol Cancer 17: 1, 2018.

17. Yang A, Wang X, Yu C, Jin Z, Wei L, Cao J, Wang Q, Zhang M, Zhang L, Zhang L, et al: microRNA-494 is a potential prognostic marker and inhibits cellular proliferation, migration and invasion by targeting SIRT1 in epithelial ovarian cancer. Oncol Lett 14 3177-3184, 2017.

18. Ma YB, Li GX, Hu JX, Liu X and Shi BM: Correlation of miR-494 expression with tumor progression and patient survival in pancreatic cancer. Genet Mol Res 14: 18153-18159, 2015.

19. Livak KJ and Schmittgen TD: Analysis of relative gene expression data using real-time quantitative PCR and the $2^{-\Delta \Delta C_{\mathrm{T}}}$ method. Methods 25: 402-408, 2001.

20. Ducassou A, Uro-Coste E, Verrelle P, Filleron T, BenouaichAmiel A, Lubrano V, Sol JC, Delisle MB, Favre G, Ken S, et al Alphavbeta3 integrin and fibroblast growth factor receptor 1 (FGFR1): Prognostic factors in a phase I-II clinical trial associating continuous administration of Tipifarnib with radiotherapy for patients with newly diagnosed glioblastoma. Eur J Cancer 49: 2161-2169, 2013
21. Bartels U, Wolff J, Gore L, Dunkel I, Gilheeney S, Allen J, Goldman S, Yalon M, Packer RJ, Korones DN, et al: Phase 2 study of safety and efficacy of nimotuzumab in pediatric patients with progressive diffuse intrinsic pontine glioma. Neuro Oncol 16: 1554-1559, 2014

22. Karremann M, Hoffmann M, Benesch M, Kwiecien R, von Bueren AO and Kramm CM: Secondary solid malignancies after high-grade glioma treatment in pediatric patients. Pediatr Hematol Oncol 32: 467-473, 2015.

23. Zhang C, Liu S, Yuan X, Hu Z, Li H, Wu M, Yuan J, Zhao Z Su J, Wang X, et al: Valproic acid promotes human glioma U87 cells apoptosis and inhibits glycogen synthase kinase-3beta through ERK/Akt signaling. Cell Physiol Biochem 39: 2173-2185, 2016

24. Wu Y, Dong L, Bao S, Wang M, Yun Y and Zhu R: FK228 augmented temozolomide sensitivity in human glioma cells by blocking PI3K/AKT/mTOR signal pathways. Biomed Pharmacother 84: 462-469, 2016.

25. Lin CJ, Chen TL, Tseng YY, Wu GJ, Hsieh MH, Lin YW and Chen RM: Honokiol induces autophagic cell death in malignant glioma through reactive oxygen species-mediated regulation of the p53/PI3K/Akt/mTOR signaling pathway. Toxicol Appl Pharmacol 304: 59-69, 2016.

26. Su S, Luo, Liu X, Liu J, Peng F, Fang C and Li B: miR-494 up-regulates the PI3K/Akt pathway via targetting PTEN and attenuates hepatic ischemia/reperfusion injury in a rat model Biosci Rep 37: 2017.

27. Li XT, Wang HZ, Wu ZW, Yang TQ, Zhao ZH, Chen GL, Xie XS, Li B, Wei YX, Huang YL, et al: $m i R-494-3 p$ regulates cellular proliferation, invasion, migration, and apoptosis by PTEN/AKT signaling in human glioblastoma cells. Cell Mol Neurobiol 35: 679-687, 2015.

28. Chen JH, Zhang P, Chen WD, Li DD, Wu XQ, Deng R, Jiao L, Li X, Ji J, Feng GK, et al: ATM-mediated PTEN phosphorylation promotes PTEN nuclear translocation and autophagy in response to DNA-damaging agents in cancer cells. Autophagy 11: 239-252, 2015.

29. Zhu H, Xie R, Liu X, Shou J, Gu W, Gu S and Che X: MicroRNA-494 improves functional recovery and inhibits apoptosis by modulating PTEN/AKT/mTOR pathway in rats after spinal cord injury. Biomed Pharmacother 92: 879-887, 2017. 